\title{
Deep-sea Scientific Investigation and Manned Submersible Technology
}

\begin{abstract}
The vast expansion of the blue ocean is always an intriguing sight and the deep sea is even more luring and mysterious. Deep down in the ocean are the largest mountain range on earth and canyons far grander than any on land. Actually, we know much less about the ocean, particularly the deep-sea floor, than about the far side of the Moon. Certainly, Chinese scientists are not satisfied with the current situation and ought to find more about it.

Breakthroughs in the deep-sea scientific investigation and manned submersible technology made by the CAS scientists have greatly boosted China's marine science and technology, and enhanced the China's ability to independently design and develop China's own innovative deep-sea devices.
\end{abstract}

\section{Deep-sea Scientific Investigation}

From August 2016 to October 2016, scientists at the CAS Institute of Deep-sea Science and Engineering (IDSSE) organized the first comprehensive scientific investigation over 10,000-meter depth in the abyss of Mariana Trench. Multiple devices went down over the 10.000-meter depth and acquired a large number of biological and environmental samples. For example. the 10.000-meter autonomous remote underwater vehicle Haidou developed by the Shenyang Institute of Automation (SIA). CAS achieved a maximum diving depth of 10.888 meters in February 2017. This feat made China the third country after Japan and the United States capable of developing a 10,000-meter unmanned underwater vehicle.

Scientists at the SIA also developed Tianya and Haijiao deep-sea landers. These deep-sea landers obtained a large number of near-bottom water samples, videos, photos, and some sediment samples, etc., and successfully captured a large number of hadal snailfishes and other large creatures. The acquired deep-sea samples may hold clues for many scientific issues

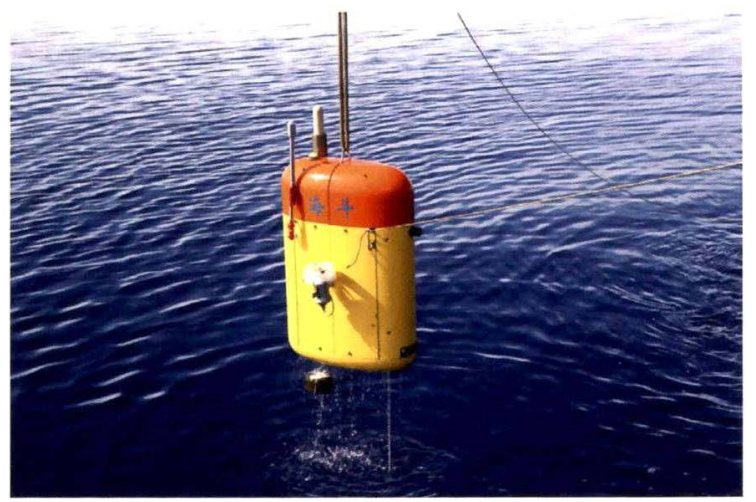

Haidou ARV in the progress of deep-sea scientific investigation. (Credit: SIA)

concerning the abyss, such as the origin and evolution of its species, its geological structure and evolution. its ecological environment, and the composition of its microbial community. These achievements also proclaimed that China's deep-sea scientific research has marched into the 10,000 -meter era.

Later in 2017, scientists at the SIA independently 
developed an underwater glider named Ilaivi. Its maximum diving depth is up to 6.329 meters. which breaks the world's record of 6.000 meters kept by the United States. Haivi underwater glider can continue to function for over 3 months. making China the second country after the United States to possess the capability to autonomous cross-quarter ocean observation.

Besides, starting from the 1980s. scientists at the CAS South China Sea Institute of Oceanology took a lead in the comprehensive scientific investigation of the Nansha Islands and its adjacent sea areas, together with 32 other units in China. This project obtained a large amount of data and information on hydrology. geology. biology. and oil and gas resources.

While enriching the basic theories of China's tropical
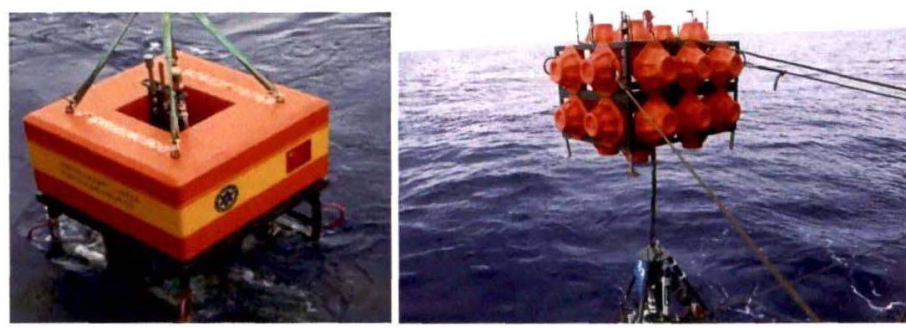

The Tianya (left) and Haijiao (right) deep-sea landers in the progress of working on site. (Credit: CAS)

marine science, it has also provided an important scientific basis for safeguarding the sovereignty and marine rights of the Nansha Islands in China. It also holds important implications for the resource exploration and environmental protection of the South China Sea.

\section{Deep-sea Manned Submersible}

To explore the deepest ocean, a deep-sea manned submersible is one of the best choices. The deep-sea submersible could quickly and accurately reach various depths for efficient exploration, scientific investigation and other kinds of deep-sea operations. Deep-sea manned submersible technology represents the top challenge in the field of deep-sea engineering. and reflects the deepsea engineering technology level of a country.

Jiaolong is China s lirst self-designed and selfdeveloped operational deep-sea manned submersible. It ranks top in terms of the diving depth among the world's deep-sea maned submersibles with operational ability. It enables (hina to be the fifth country in the world after the United States, France, Russia and Japan to master the technology of deep-sea manned diving. In the world's manned submersibles, Jiaolong belongs to the first echelon up to date.

CAS scientists at the Institute of Acoustics (IOA) and
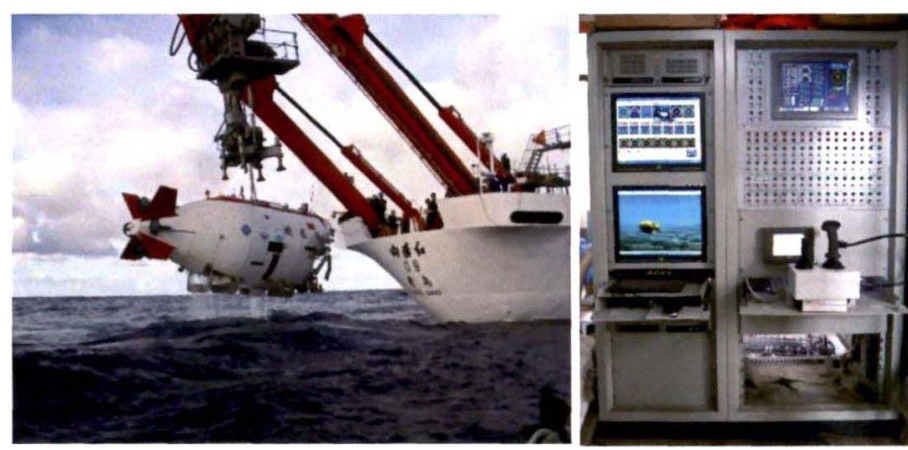

China's manned submersible Jiaolong (left) and its control and simulation platform (right). (Credit: CAS)

the Shenyang Institute of Automation (SIA) designed two key parts of Jiaolong. the acoustic system and the control system. which won the 2013 Outstanding Scientific and Technological Achievement Award of the Chinese Academy of Sciences.

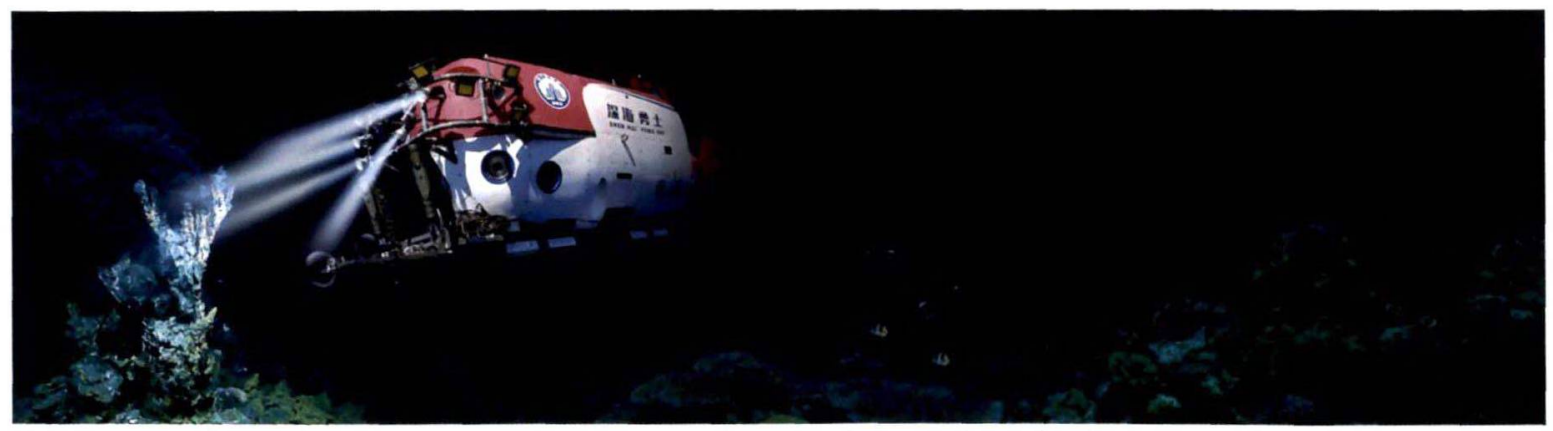

An art illustration of the Deep-sea Warrior manned submersible exploring in the deep sea. (Credit: IDSSE) 
Jiaolong set a record for the active operational manned submersible diving with a maximum dive of 7.062 meters on June 27, 2012, marking that China's manned deep diving technology has achieved the world's leading level. In fact, the current record for the deepest manned dive in the world was made by the US Trieste. In 1960, it dived to 10,911 meters, but it is an adventure type, which is relatively simple in function - drop down. pull up and basically do nothing operational.

Compared with other deep-sea manned submersibles, apart from its deepest diving depth, Jiaolong has more advanced communication technology: it has a very advanced underwater acoustic communication system that enables the cross-talking between the depot ship and the submersible. Pulling a cable from the water surface to the submersible over a long distance of seven kilometers is impractical. Besides, the radio would be blocked by the seawater. It leaves no choice but to rely entirely on underwater acoustic communication to turn the information to be transmitted into sound. After it is transmitted to the water surface, the sound is transformed back into the encoded information. This underwater acoustic communication system can transmit the data collected by many sensors in the submersible to the water surface. The water surface and underwater can communicate through text, and even send photos. In spite of its many refreshed technical and diving depth records, it has to be acknowledged that the submersible still relies on foreign countries for some key technologies including materials.

The China's second deep-sea manned submersible named Shenhai Yongshi, or Deep-sea IVarrior, was developed by CAS scientists at the Institute of Acoustics, Shenyang Institute of Automation, and Technical Institute of Physics and Chemistry. Deep-sea Warrior owns China's independent intellectual property rights, and its localization rate reaches $9.5 \%$.

In its first manned deep dive test led by the CAS Institute of Deep-sea Science and Engineering (IDSSE) in August 2017, Deep-sea Warrior completed 28 deep dives with a maximum depth of 4,534 meters, which basically allows a thorough investigation of China's

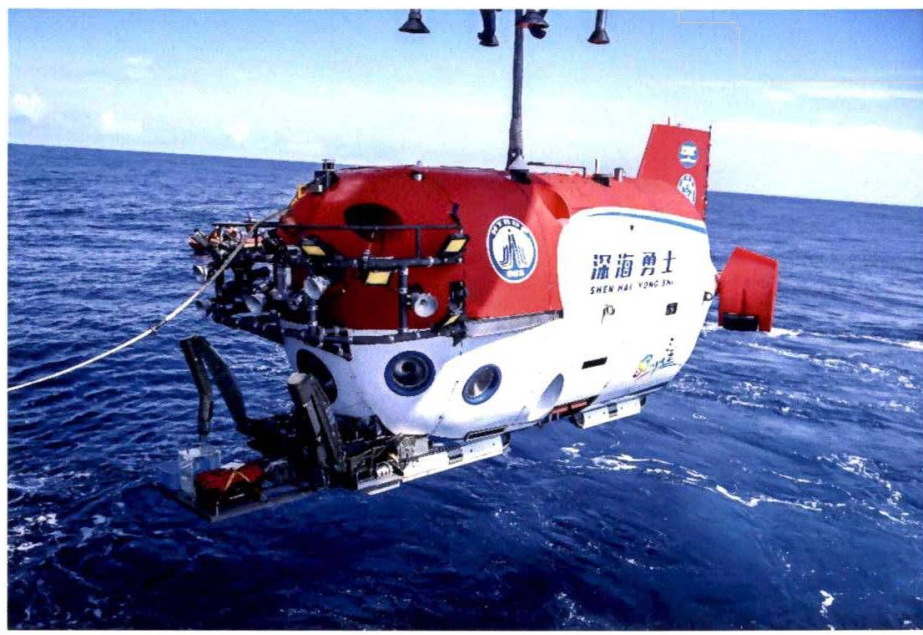

The Deep-sea Warrior deep-sea manned submersible. (Credit: IDSSE)

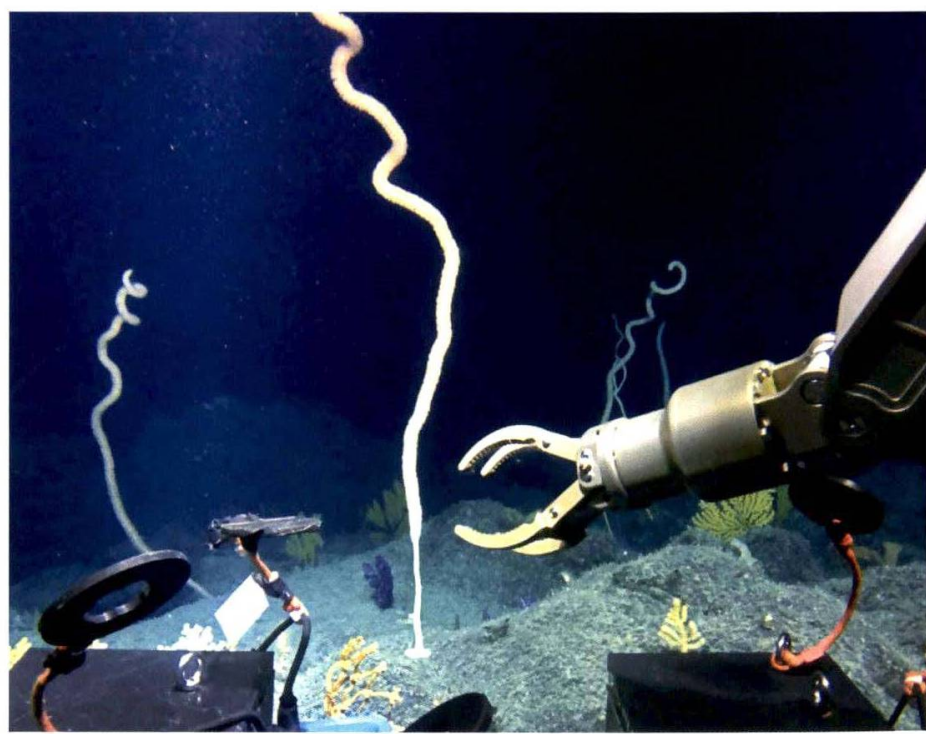

The camera view of the Deep-sea Warrior at working on the sea floor. (Credit: IDSSE)

main sea areas and international sea areas. In addition, hot topics in international deep-sea science, such as submarine hydrothermal sulfide and subsea cold springs (approximately 3,000 meters in depth below the sea surface) are also within the dive range. Deep-sea Warrior has been used for the hydrothermal and geological studies of the sea floor, underwater archaeology, rescue, search and so on. 